\title{
Radiation Sterilization
}

National Cancer Institute

\section{Source}

National Cancer Institute. Radiation Sterilization. NCI Thesaurus. Code C101715.

A sterilization process that uses radiation such as electron beams, X-rays, gamma rays, or subatomic particles to kill microorganisms. 